\title{
Application of Carbon Nanotubes Modified Coatings for the Determination of Amphetamines by In-Tube Solid-Phase Microextraction and Capillary Liquid Chromatography
}

\author{
Ana Isabel Argente-García, Yolanda Moliner-Martínez, Esther López-García, Pilar Campíns-Falcó \\ and Rosa Herráez-Hernández * \\ Department of Analytical Chemistry, University of Valencia, Dr. Moliner 50, Burjassot, Valencia 46100, Spain; \\ arai@uv.es (A.I.A.-G.); yolanda.moliner@uv.es (Y.M.-M.); estloga3@uv.es (E.L.-G.); pilar.campins@uv.es (P.C.-F.) \\ * Correspondence: rosa.herraez@uv.es; Tel.: +34-96-3544978; Fax: +34-96-3544436
}

Academic Editor: Victoria F. Samanidou

Received: 17 December 2015; Accepted: 6 February 2016; Published: 1 March 2016

\begin{abstract}
In this study, polydimethylsiloxane (PDMS)-coated capillary columns (TRB-5 and TRB-35), both unmodified and functionalized with single-wall carbon nanotubes (SWCNTs) or multiwall carbon nanotubes (MWCNTs), have been tested and compared for the extraction of amphetamine (AMP), methamphetamine (MET) and ephedrine (EPE) by in-tube solid-phase microextraction (IT-SPME). Prior to their extraction, the analytes were derivatized with the fluorogenic reagent 9-fluorenylmethyl chloroformate (FMOC). For separation and detection capillary chromatography with fluorimetric detection has been used. The presence of carbon nanotubes in the extractive coatings enhanced the extraction efficiencies and also significantly improved the chromatographic profiles, thus resulting in a reliable option for the analysis of these drugs. As an example of application, a new method is proposed for the analysis of the tested amphetamines in oral fluid using a TRB-35 capillary column functionalized with MWCNTs. The proposed conditions provided suitable selectivity and reproducibility $(\mathrm{CV} \leqslant 6 \%, n=3)$ at low $\mu \mathrm{g} / \mathrm{mL}$ levels, and limits of detection of $0.5-0.8 \mu \mathrm{g} / \mathrm{mL}$.
\end{abstract}

Keywords: carbon nanotubes (CNTs); in-tube solid-phase microextraction (IT-SPME); derivatization; amphetamines; capillary liquid chromatography

\section{Introduction}

Among the novel microextraction techniques developed in the last decades, in-tube solid-phase microextraction (IT-SPME) has emerged as one of the most attractive options, as demonstrated by the increasing number of publications that use it for the analysis of a variety of analytes and matrices [1]. IT-SPME typically uses a polymeric-coated capillary column coupled to a liquid chromatograph. The target compounds can be extracted by repeated draw/injection cycles of the samples using a programmable sample injector until the analytes reach partition equilibrium between the coating of the capillary and the sample. Sample volumes are typically in the 100-500 $\mu \mathrm{L}$ range, although they are usually mixed with an aliquot of an organic solvent or a buffer before the extraction. Alternatively, the extractive capillary can be used as the loop of the injector valve (in-valve IT-SPME), and a volume of sample as large as necessary (up to several $\mathrm{mL}$ ) is passed through the capillary until the amount of analytes extracted is sufficient to reach the required analytical responses. The former option has been extensively used for the analysis of drugs and biomarkers in biological fluids, whereas the in-valve IT-SPME microextraction approach is particularly well-suited for the extraction of traces of organic pollutants from water samples [1-3]. 
In IT-SPME the extraction efficiency depends on a variety of factors such as the capillary dimensions, and the type and thickness of the extractive coating. Although segments of commercially available open tubular capillary columns such as those used in gas chromatography (GC) can be used for many applications, improved selectivity and extraction efficiency can be expected through the development of new extractive phases [4,5]. Examples of alternative phases developed for IT-SPME are polypyrrole-based coatings, restricted access materials, immunosorbents or molecularly imprinted polymers (MIPs), as well as monolithic packings [6].

Nanostructured coatings are becoming popular in the context of extraction and microextraction mainly because they offer high surface-to-volume ratios, thus increasing the extraction efficiencies. In this sense, the employment of sorbents with carbon nanotubes (CNTs) appears as one of the most attractive options. CNTs have been extensively tested as sorbents for solid phase extraction (SPE) and fiber SPME because they can interact with a variety of compounds through different mechanisms such as hydrogen bonding, $\pi-\pi$ stacking interactions, electrostatic and van der Waals forces, and hydrophobic interactions [7,8]. However, only a few studies have been reported on the utilization CNTs modified phases for IT-SPME. Liu et al. immobilized - $\mathrm{COOH}$ functionalized MWCNTs onto the external surface of a fused silica fiber using epoxy resin glue. The fibre was then inserted into a PEEK tube which was used as the injection loop in an in-valve IT-SPME configuration. The proposed device was applied to the extraction of aniline compounds from water [9]. In recent studies, we have reported the use of different polydimethylsiloxane (PDMS)-based capillary columns modified covalently with CNTs. The new coatings provided improved efficiencies for the extraction of some pollutants from water samples [10,11].

Enhancing the extraction capability of the capillary coating may be the key factor to extend the applicability of in-valve IT-SPME to those analytical problems in which the volume of sample is limited. A typical example is the identification and determination of drugs in oral fluid. Although there are several methods available for drugs in plasma and urine samples, there is an increasing interest in the analysis of alternative biological matrices such oral fluid or sweat [12,13]. In particular, oral fluid is the matrix preferred for the investigation of illicit drug consumption from drivers suspected for driving under the influence of drugs. This is because sample collection is non-invasive and it can be done under supervision. Therefore, the risk of sample adulteration is minimized. Moreover, drug concentrations in oral fluid are generally higher than in other biological fluids, especially after oral intake. The main difficulty encountered in the analysis of these fluids is the low amount of sample available. Thus, most of the analytical schemes proposed for the analysis of plasma and urine can hardly be applied to these alternative fluids.

In the present study, the extraction capabilities of CNTs modified PDMS-based capillaries for amphetamines have been explored. Different PDMS-based extractive capillaries both unmodified and modified with CNTs have been investigated. As indicated above, these coatings have been previously applied to the extraction of organic pollutants from water samples $[10,11]$. In the present work, we have evaluated for the first time the potential utility of such extractive phases in order to extend the IT-SPME methodology to drug analysis in oral fluid. To our knowledge CNTs modified coating have never been used for the extraction of drugs by in-valve IT-SPME. Amphetamine (AMP), methamphetamine (MET) and ephedrine (EPE) have been selected as model compounds because, according to the literature, amphetamines (including amphetamine-derived designer drugs) are among the most frequently detected drugs in oral fluid from drivers [13]. Routine analysis of amphetamines in biological samples are performed on a daily basis in clinical, forensic and toxicological laboratories [14].

Immunoassays are generally used for screening tests, whereas chromatographic methods are required for quantitative purposes. Chromatographic procedures involve extensive sample treatment for analyte isolation and preconcentration [12,13]. The determination of amphetamines by liquid chromatography (LC) usually entails a previous chemical derivatization to make the analytes more amenable for chromatography and/or to enhance the sensitivity. According to the results obtained in previous studies, in the present work the fluorogenic reagent FMOC has been selected for derivatization 
(see Figure 1) $[15,16]$. The amphetamine derivatives formed have been processed by in-valve IT-SPME coupled online to capillary liquid chromatography with fluorimetric detection. The analytical performance and possible applications of the proposed approach are discussed.<smiles>[R]NC(C)C([R])c1ccccc1</smiles>

Analyte
FMOC<smiles>[R]NC(=O)C(C)C([R])c1ccccc1</smiles>

Analyte-FMOC derivative

\begin{tabular}{lll}
\hline & $\mathbf{R}_{\mathbf{1}}$ & $\mathbf{R}_{\mathbf{2}}$ \\
\hline ANF & $\mathrm{H}$ & $\mathrm{H}$ \\
MET & $\mathrm{CH}_{3}$ & $\mathrm{H}$ \\
EPE & $\mathrm{CH}_{3}$ & $\mathrm{OH}$ \\
\hline
\end{tabular}

Figure 1. Scheme of the reaction between FMOC and the amphetamines tested.

\section{Experimental Section}

\subsection{Reagents and Solutions}

All the reagents were of analytical grade. SWCNTs, MWCNTs, 3-aminopropyl triorthoxysilane (APTS), 1,3-dicyclohexylcarbodiimide, glutaraldehyde, AMP sulphate, MET hydrochloride and EPE hydrochloride were obtained from Sigma (St. Louis, MO, USA), and FMOC was purchased from Aldrich (Stenheim, Germany). Sodium hydrogen carbonate was purchased Probus (Badalona, Spain). Acetonitrile was of HPLC grade (Romil, Cambridge, UK); methanol and acetone were purchased from Scharlau (Barcelona, Spain). Sodium hydroxide, nitric acid $(60 \%)$, sulphuric acid $(98 \%)$ and dimethylformamide were purchased from Panreac (Barcelona, Spain).

Stock standard solutions of AMP, MET and EPE $(1000.0 \mu \mathrm{g} / \mathrm{mL})$ were prepared in water. Working solutions of these compounds were prepared by dilution of the stock solutions with water. Stocks solutions of FMOC $(10 \mathrm{mM})$ were prepared weekly by dissolving the pure compound in acetonitrile. Solutions used for derivatization $(0.1 \mathrm{mM})$ were prepared daily by dilution of the $10 \mathrm{mM}$ FMOC stock solutions with acetonitrile. The hydrogencarbonate buffer $(4 \%, w / v)$ was prepared by dissolving the appropriate amount of sodium hydrogen carbonate in water, and then by adjusting the $\mathrm{pH}$ to 10.6 with $5 \mathrm{M} \mathrm{NaOH}$.

Ultrapure water was obtained from a Nanopure II (Sybron, Barnstead, UK) system. All solutions were stored in the dark at $4{ }^{\circ} \mathrm{C}$.

\subsection{Apparatus and Chromatographic Conditions}

The chromatographic system consisted of an isocratic capillary pump, a high-pressure six-port valve (Reodyne, Rohnert Park, CA, USA), a LC-Net II/ADC interface and a fluorescence detector (Micro 21PU-01, Jasco Corporation, Tokyo, Japan). The detector was coupled to a data system (Jasco ChromNAV Chromatography Data System) (Jasco Corparation, Tokyo, Japan) for data acquisition and calculation. The excitation and emission wavelengths were $285 \mathrm{~nm}$ and $320 \mathrm{~nm}$, 
respectively. A Zorbax SB-C18 ( $35 \mathrm{~mm} \times 0.5 \mathrm{~mm}$ i.d., $3.5 \mu \mathrm{m}$ ) column (Agilent, Waldbronn, Germany) was used for the separation of the analytes. The mobile-phase was a mixture of acetonitrile-water, and the flow rate was $20 \mu \mathrm{L} / \mathrm{min}$.

\subsection{IT-SPME}

The set-up used in the present study corresponds to that developed for in-valve IT-SPME [1,2]. The stainless steel loop of the injection valve was replaced by the extractive capillary. In this study segments of PDMS-based open tubular columns $(0.32 \mathrm{~mm}$ i.d., $3 \mu \mathrm{m}$ coating thickness) were used as extractive capillaries. The columns tested were TRB-35 (35\% diphenyl-65\% polydimethylsiloxane) and TRB-5 (5\% diphenyl-95\% polydimethylsiloxane), both purchased from Teknokroma (Barcelona, Spain). Segments of 30-50 cm these columns were directly tested for IT-SPME or functionalized with SWCNTs or MWCNTs.

The procedures used for the functionalization of the columns were extensively described in [10]. Briefly, the $0.025 \mathrm{~g}$ of SWCNTs were previously carboxylated with $5 \mathrm{~mL}$ of a mixture of sulfuric and nitric acids $(3: 1, v / v)$; MWCNTs were carboxylated by adding $80 \mathrm{~mL}$ of a mixture of sulfuric and nitric acids $(3: 1, v / v)$ to $0.020 \mathrm{~g}$ of MWCNTs; The PDMS columns were activated by passing successively $2 \mathrm{M} \mathrm{NaOH}(24 \mathrm{~h})$, water $(5 \mathrm{~min}),(2 \%, v / v)$ APTS in anhydrous acetone (15 min), water (5min), methanol (5 min) and 10\% glutaraldehyde prepared in $50 \mathrm{mM}$ borate buffer of $\mathrm{pH} 9.0$ (10 min). Then, suspensions of the carboxylated CNTs (c-CNTs) $5 \mathrm{mg} / \mathrm{mL}$ prepared in dimethylformamide containing 1,3-dicyclohexylcarbodiimide were passed through the activated capillaries for $30 \mathrm{~min}$ in order to immobilize the c-CNTs into the PDMS-based coating. Finally, unreacted c-CNTs were eliminated by flushing water through the capillaries. The total time required for preparation of the capillaries after activation of the PDMS phase was about $2 \mathrm{~h}$. Capillary connections to the valve were facilitated by the use of $2.5 \mathrm{~cm}$ sleeve of $1 / 16$ in. polyether ether ketone (PEEK) tubing (Teknokroma); $1 / 16$ in PEEK nuts and ferrules (Teknokroma) were used to complete the connections.

Aliquots of the solutions to be processed $(20-50 \mu \mathrm{L})$ were manually loaded into the extractive capillary by means of a $100 \mu \mathrm{L}$-precision syringe. Next, $20 \mu \mathrm{L}$ of water were flushed through the capillary in order to eliminate the solution remaining into it. Finally, the valve was manually rotated so the analytes were desorbed from the coating of the extractive capillary with the mobile-phase, and transferred to the analytical column for separation and detection.

\subsection{Derivatization of the Amphetamines}

Conditions for the derivatization of the tested amphetamines were selected according to previous works [14,15]. Aliquots of the standard solutions of the amphetamines $(125 \mu \mathrm{L})$ were placed into $2 \mathrm{~mL}$ glass vials, and mixed with $125 \mu \mathrm{L}$ of carbonate buffer and with $250 \mu \mathrm{L}$ of a solution of the derivatization reagent $(0.1 \mathrm{mM}$ of FMOC). After a reaction time of $5.0 \mathrm{~min}, 50 \mu \mathrm{L}$ of the resulting mixture were loaded into the extractive capillary of the IT-SPME device.

Each sample was derivatized in duplicate and all assays were carried out at ambient temperature.

\subsection{Analysis of Real Samples}

Samples (standard solutions of the analytes or oral fluid) were placed in 2-mL glass vials; then, one of the tips of a cotton swab was immersed into the sample, so that the cotton was totally wetted by the sample. The swab was then removed and introduced into a 2-mL glass vial containing $250 \mu \mathrm{L}$ of the carbonate buffer and $250 \mu \mathrm{L}$ of the $0.1 \mathrm{mM}$ FMOC solutions. The swab was left inside the derivatization solution for $5 \mathrm{~min}$, so the analytes passed from the cotton to the solution and the reaction occurred. After the reaction period, the swab was discarded, and an aliquot of the solution was removed by means of a $100 \mu \mathrm{L}$ syringe and loaded into the IT-SPME capillary. Drug-free oral fluid samples were obtained from volunteers after informed consent. Cotton swaps were purchased from a local market; the amount of cotton on each tip was of about $0.03 \mathrm{~g}$. 
Unless otherwise stated, each sample was derivatized in duplicate and all assays were carried out at ambient temperature.

\section{Results and Discussion}

\subsection{Optimization of the IT-SPME and Chromatographic Conditions}

Initially, experiments were carried out in order to optimize conditions for the transfer of the FMOC derivatives from the extractive capillary of the IT-SPME device to the analytical column, as well as for the subsequent chromatographic separation. In these studies, unmodified capillaries were used for IT-SPME. The mobile-phase was a mixture acetonitrile-water and flow-rate was $20 \mu \mathrm{L} / \mathrm{min}$.

When IT-SPME is combined on-line with capillary liquid chromatography, peak widths of the analytes are significantly higher than those typically achieved by conventional capillary chromatography, which is due to the inclusion of the extractive capillary in the chromatography system. A reduction of the extractive capillary dimensions would improve the resolution, but at the expense of a lower sensitivity (lower amount of analyte extracted). On the other hand, under a conventional capillary chromatographic scheme, the resolution could be improved by using lower mobile-phase flow rates. However, when an IT-SPME device is added to the chromatographic system, a reduction in the mobile-phase flow rate would increase the time of residence of the analytes in the extractive capillary causing extra peak broadening [17]. In other words, when in-valve IT-SPME is coupled on-line to capillary liquid chromatography, a compromise has to be reached between optimal conditions for the IT-SPME and for the chromatographic separation. In the present study, conditions were selected to achieve suitable separation of the three analytes of interest in the minimum time of analysis. Indeed, extending the proposed methodology to other amphetamine-like compounds would require different separation conditions (for example, a longer column).

Two mobile-phase compositions were tested, 50:50 and 70:30 acetonitrile:water $(v / v)$. The results obtained demonstrated that both compositions were adequate to desorb the FMOC derivatives from the extractive capillary. However, best chromatographic separation was achieved by using a mixture acetonitrile:water 70:30 ( $v / v$ ) (see Figure S1), which was the eluent composition selected for further work. The dead times observed in the chromatograms were higher than those expected for a conventional capillary chromatographic separation due to the inclusion of the extractive device. It has to be noted that the EPE-FMOC derivative eluted on the tail of the FMOC peak; for this reason, peak areas for this analyte were calculated throughout the study by the tangent skimming integration method.

On the other hand, the effect of the capillary length on the analytical responses (peak areas) was evaluated by processing solutions of the same concentration of amphetamines with TRB-35 capillaries of 30, 40 and $50 \mathrm{~cm}$. In principle, an increment in the capillary length increases the surface area available for extraction, and thus, the amount of analyte extracted also increased. A slight increment on the analyte peak areas was observed when extending the capillary length from $30 \mathrm{~cm}$ to $40 \mathrm{~cm}$. Unfortunately, the amount of unwanted products extracted also increases. In the present case, the chromatographic profile observed for an extractive capillary of 50-cm length was unsuitable due to the large amount of unreacted FMOC extracted and transferred to the analytical column (see Figure S2). Consequently, capillaries longer than $40 \mathrm{~cm}$ were not considered for further assays.

\subsection{Evaluation of the Extraction Efficiencies}

The extraction efficiency was evaluated for unmodified TRB- 5 and TRB-35 capillaries, as well as for TRB-5 capillaries after their functionalization with c-SWCNTs, and TRB-35 capillaries functionalized with c-SWCNTs or with c-MWCNTs. The length of TRB-5 capillaries was $36 \mathrm{~cm}$, whereas all TRB-35 capillaries were $40-\mathrm{cm}$ length. The mean peak areas obtained for the tested capillaries are depicted in Figure 2; it has to be mentioned that AMP and MET peak areas were calculated by a valey-to-valey integration approach. 


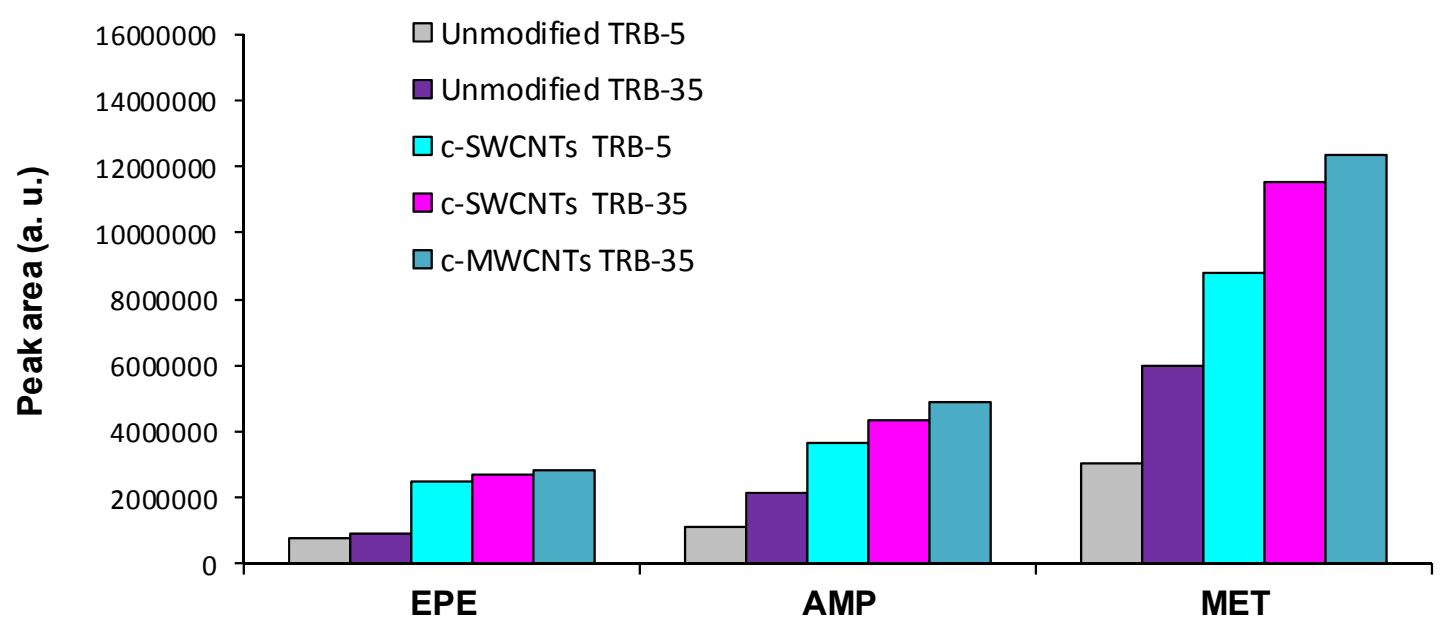

Figure 2. Peak areas obtained for a standard solution of the amphetamines tested $(8.0 \mu \mathrm{g} / \mathrm{mL}$, each) with different extractive capillaries: unmodified TRB-5 $(36 \mathrm{~cm})$, unmodified TRB-35 $(40 \mathrm{~cm})$, c-SWCNTs functionalized TRB-5 $(36 \mathrm{~cm})$, c-SWCNTs functionalized TRB-35 $(40 \mathrm{~cm})$ and c-MWCNTs functionalized TRB-35 $(40 \mathrm{~cm})$. For other experimental details, see text.

In order to compare the extraction efficiencies, the preconcentration rates (established as the peak area ratios between two given capillaries) were also calculated [10]. The results are summarized in Table 1.

Table 1. Preconcentration rates (PRs) values found for the extractive capillaries tested.

\begin{tabular}{cccc}
\hline Capillaries Compared & \multicolumn{3}{c}{ PR } \\
\cline { 2 - 4 } & EPE & AMP & MET \\
\hline Unmodified TRB-35/Unmodified TRB-5 & 1.2 & 2.0 & 2.0 \\
c-SWCNT functionalized TRB-5/Unmodified TRB-5 & 3.3 & 3.3 & 2.9 \\
c-SWCNT functionalized TRB-35/Unmodified TRB-35 & 2.9 & 2.2 & 1.9 \\
c-SWCNT functionalized TRB-35/c-SWCNTs functionalized TRB-5 & 1.1 & 1.2 & 1.3 \\
c-MWCNT- functionalized TRB-35/c-SWCNTs functionalized TRB-35 & 1.0 & 1.1 & 1.1 \\
\hline
\end{tabular}

First, the solutions containing the three amphetamines were assayed onto the two unmodified capillaries tested. Higher analytical responses for the three analytes were obtained with the TRB-35 phase, as it can be seen in Table 1. It can be deduced that a higher percentage of diphenyl groups in the extractive phase lead to higher extraction rates. This suggests that the extraction involves $\pi-\pi$ interactions with the FMOC derivatives, which is consistent with the fact that the extracted compounds possess three aromatic rings (see Figure 1).

The effect of the presence of CNTs in the extractive coating was investigated for the TRB-5 and TRB-35 capillaries. In Figure 3 are depicted the chromatograms obtained for an unmodified TRB-5 capillary and the same capillary functionalized with c-SWCNTs. As observed, the presence of c-SWCNTs in the extractive phase affected not only the peak areas of the FMOC derivatives, but also their retention times. Higher retention times were observed with the c-SWCNTs coated phase, which indicates that the interaction between the extractive phase and the FMOC derivatives is stronger in the presence of CNTs, most probably by a $\pi-\pi$ mechanism [10]. This can also explain the higher peak areas observed with the c-SWCNTs functionalized coating (see Figure 2); the analyte responses were about three times higher with the modified coating (see Table 1). 


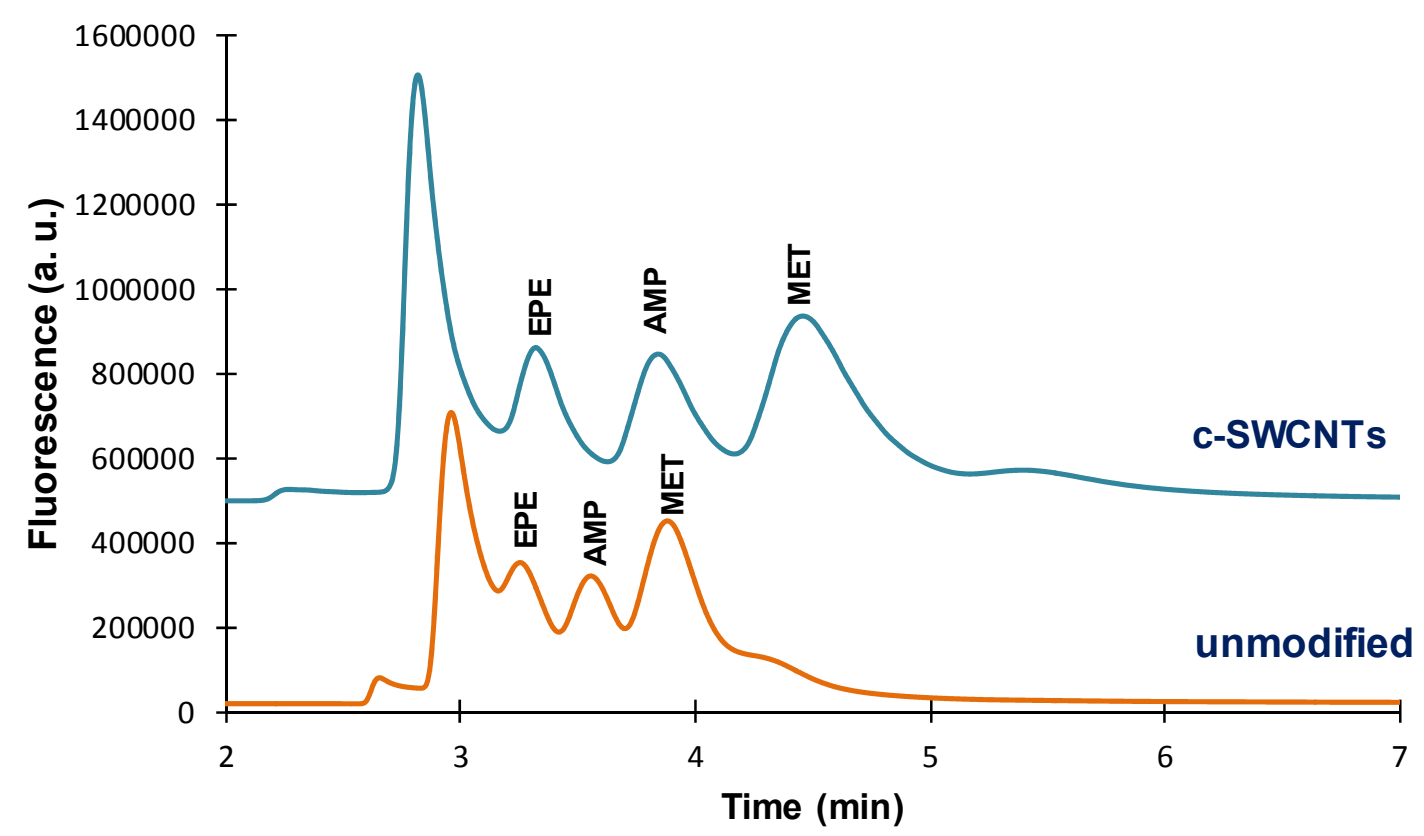

Figure 3. Chromatograms obtained for a standard solution of the amphetamines tested $(8.0 \mu \mathrm{g} / \mathrm{mL}$, each) with the unmodified TRB- 5 and the c-MWCNTs functionalized TRB- 5 capillaries. For other experimental details, see text.

Similar results were observed for the PDMS-TRB-35 capillaries. This is illustrated in Figure 4, which shows the chromatograms obtained for unmodified TRB-35 capillary and the same capillary after the introduction of c-MWCNTs. However, the increment in the analytical signals was more moderate than with the TRB-5 capillaries, especially for AMP and MET (Table 1). It is interesting to note that, although the retention times slightly increased in the presence of c-MWCNTs, the chromatographic peaks are narrower. As a result, not only the sensitivity but also the resolution improved with the introduction of the CNTs.

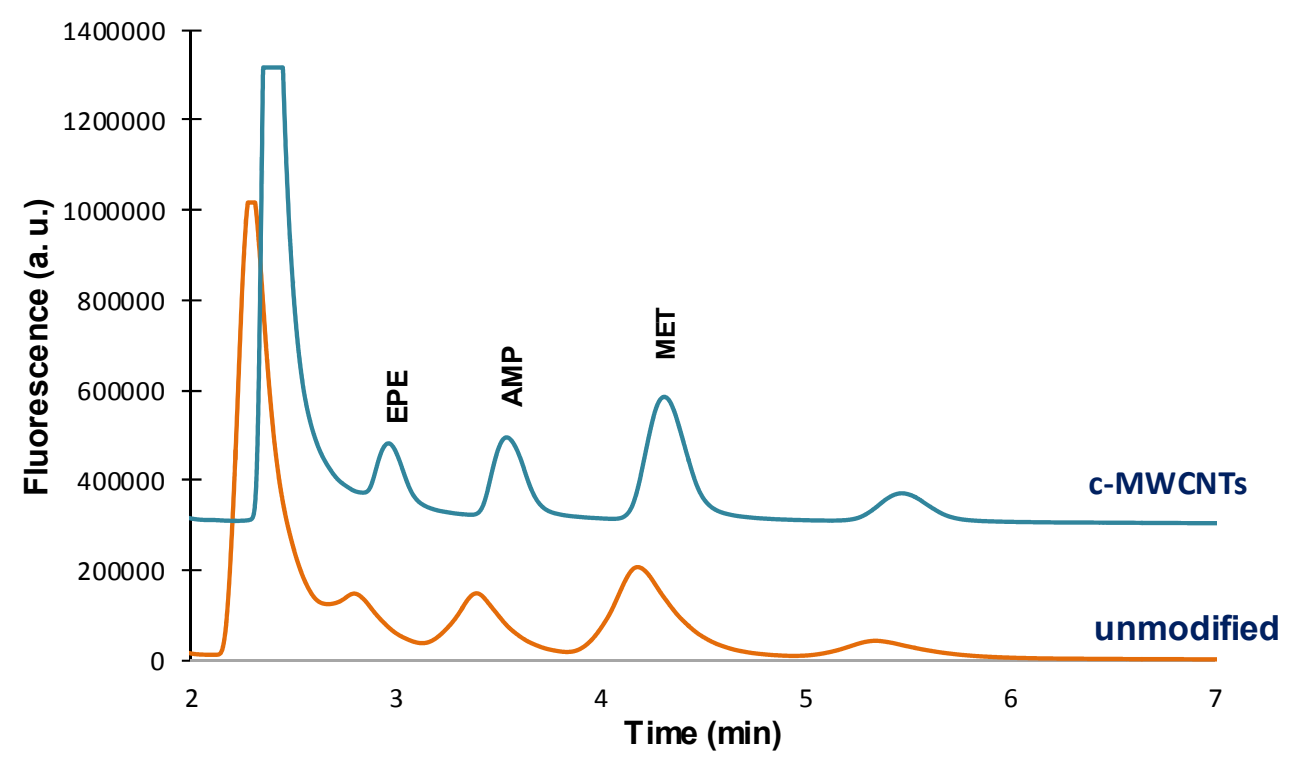

Figure 4. Chromatograms obtained for a standard solution of the amphetamines tested $(8.0 \mu \mathrm{g} / \mathrm{mL}$, each) with the unmodified TRB-35 and the c-MWCNTs functionalized TRB-35 capillaries. For other experimental details, see text. 
On the other hand, the effect of the type of CNTs on the extractive phase was evaluated for a TRB-35 capillary. Figure 5 shows that both c-SWCNTs and c-MWCNTs modified coatings provided similar chromatographic profiles, although the peak areas were slightly higher for the c-MWCNTs functionalized capillary. The chromatographic profiles obtained when using the TRB-35 capillaries were better than those observed with the TRB-5 capillaries (Figures 3-5).

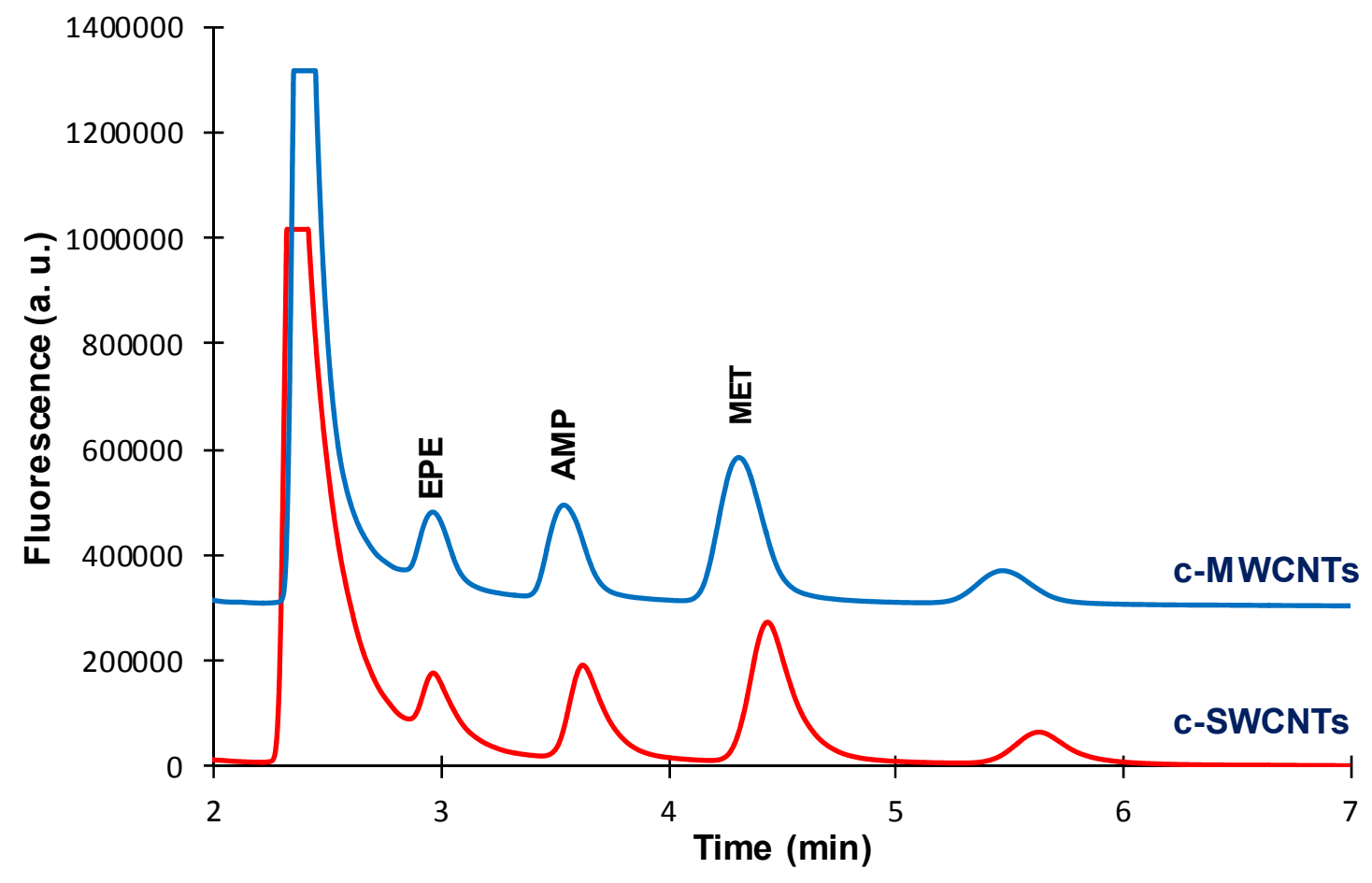

Figure 5. Chromatograms obtained for a standard solution of the amphetamines tested $(8.0 \mu \mathrm{g} / \mathrm{mL}$, each) with the c-SWCNTs and the c-MWCNTs functionalized TRB-35 capillaries. For other experimental details, see text.

Finally, the loading capacity was examined by processing solutions of the tested analytes at concentrations ranging from 2.0 to $10.0 \mu \mathrm{g} / \mathrm{mL}$ with different capillaries. The results are depicted in Figure 6. As it can be deduced from this figure, a linear relation between concentration and peaks areas was observed within the tested concentration interval with the TRB-35 columns. However, poor linearity was observed for the TRB- 5 columns regardless the presence of CNTs in the extractive coating. For the TRB- 5 capillaries, better correlation was observed between peak areas and concentration within the $2.0-8.0 \mu \mathrm{g} / \mathrm{mL}$ range (see Table S1), which indicates that their loading capacity was lower than that of the TRB-35 capillaries.

Taking into account the absolute peak areas and chromatographic profiles, as well as the linear working interval, a c-MWCNTs coated TRB-35 capillary of $40 \mathrm{~cm}$ length was selected as the best option for further experiments. 

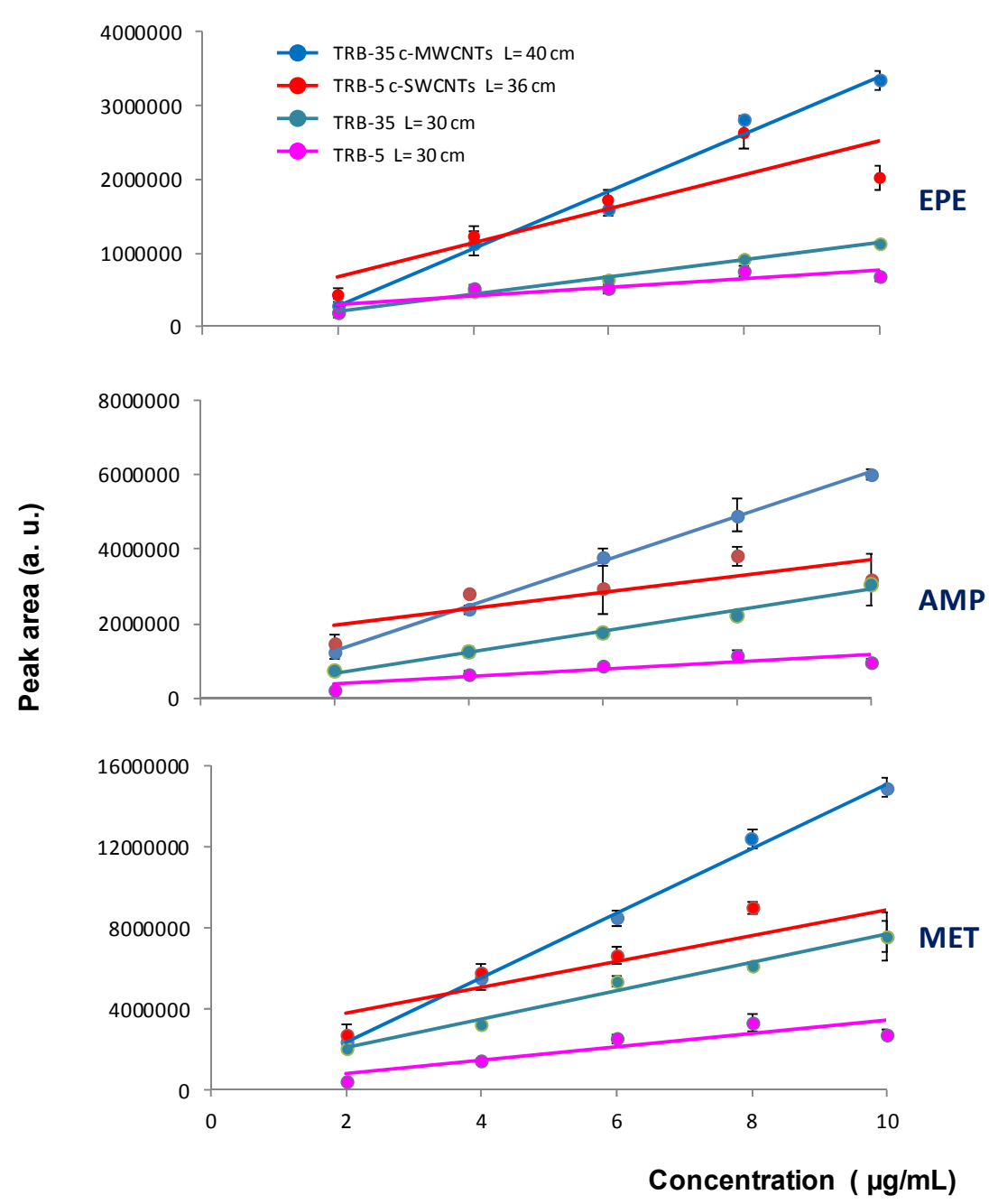

Figure 6. Peak areas obtained for different concentrations of the amphetamines tested within the $2.0-10.0 \mu \mathrm{g} / \mathrm{mL}$ concentration range with different extractive capillaries. For other experimental details, see text.

\subsection{Application to the Quantification of Amphetamines in Oral Fluid Samples}

In the analysis of drugs in oral fluid, samples are usually collected by spitting or by using sweat wipes, sponges or swabs $[12,13]$. Owing to its high viscosity the direct loading of oral fluid into the IT-SPME capillary was unsuitable, even after dilution with the buffer and FMOC solutions. For this reason, in the present study cotton swabs were used to collect oral fluid. In order to simplify the analytical procedure and to avoid excessive dilution of the analytes, the swabs were directly immersed into vials containing the derivatization solution. In such a way, the analytes were simultaneously extracted from the swabs and derivatized. Finally, aliquots of the resulting solutions were removed from the vials and processed by IT-SPME with a c-MWCNTs coated TRB-35 capillary. The reliability of the proposed approach was first tested with standard solutions of the analytes.

Preliminary experiments were carried out to estimate the volume of the sample that could be absorbed with the swabs. Volumes of the working solutions ranging from 50 to $200 \mu \mathrm{L}$ were introduced into 2-mL glass vials, and then swabs were put into contact with these solutions. It was found that the swabs used in the present study absorbed about $125 \mu \mathrm{L}$ of the samples. According to this observation, quantitative studies were carried out by placing aliquots $125 \mu \mathrm{L}$ of the standard solutions containing the amphetamines in glass vials; then, the tips of the swabs were put into contact with the working solutions until the liquid was totally absorbed. Next, the swabs were immersed into 
another vial which contained the derivatization solution ( $250 \mu \mathrm{L}$ of the carbonate buffer and $250 \mu \mathrm{L}$ of the $0.1 \mathrm{mM}$ FMOC solutions). Finally, aliquots of the resulting solutions were processed by IT-SPME and chromatographed.

The effect of volume of the extracts loaded into the IT-SPME capillary was tested within the 10-50 $\mu \mathrm{L}$ interval. A volume of $20 \mu \mathrm{L}$ was found to be the best option, as higher volumes did not significantly increase the signal, probably due to the high percentage of acetonitrile in the extracts, whereas the peak corresponding to the excess of FMOC interfered with the measurement of the FMOC-EPE peak. Under such conditions, good linearity and reproducibility was found within the tested concentration intervals $(1.0-10.0 \mu \mathrm{g} / \mathrm{mL}$ for AMP and MET and $2.0-10.0 \mu \mathrm{g} / \mathrm{mL}$ for EPE) (see Table 2).

Table 2. Linearity and reproducibility achieved with the proposed extraction/derivatization-ITSPME-capillary liquid chromatographic method for AMP, MET and EPE within the 1.0-10.0 $\mu \mathrm{g} / \mathrm{mL}$ (2.0-10.0 $\mathrm{\mu g} / \mathrm{mL}$ for EPE) concentration interval (values obtained with the c-MWCNTs coated TRB-35 capillary of $40 \mathrm{~cm}$ length).

\begin{tabular}{|c|c|c|c|c|}
\hline \multirow{2}{*}{ Compound } & \multicolumn{3}{|c|}{ Linearity, $y=a x+b(n=8) *$} & \multirow{2}{*}{$\begin{array}{c}\text { Reproducibility }{ }^{* *}, \\
\text { Relative Standard } \\
\text { Deviation (RSD) }(\%)(n=3)\end{array}$} \\
\hline & $a$ & $b$ & $R^{2}$ & \\
\hline $\mathrm{EPE}$ & $(229 \pm 8) \times 10^{3}$ & $(-18 \pm 4) \times 10^{4}$ & 0.991 & 5 \\
\hline AMP & $(402 \pm 15) \times 10^{3}$ & $(-39 \pm 8) \times 10^{4}$ & 0.991 & 3 \\
\hline MET & $(606 \pm 22) \times 10^{3}$ & $(-46 \pm 11) \times 10^{4}$ & 0.991 & 3 \\
\hline
\end{tabular}

${ }^{*}$ Established from four concentrations assayed in duplicate; ${ }^{* *}$ established at a concentration of $4.0 \mu \mathrm{g} / \mathrm{mL}$.

According to the above results, the conditions finally selected for the analysis of amphetamines in oral fluid were those indicated in Figure 7. A small peak was observed at a retention time slightly lower than that of the EPE-FMOC derivative. This peak was not detected when effecting the derivatization in solution; thus, this peak was due to a compound present in the cotton. Nevertheless, it could be differentiated from the peak corresponding to the EPE derivative (see Figure S3).
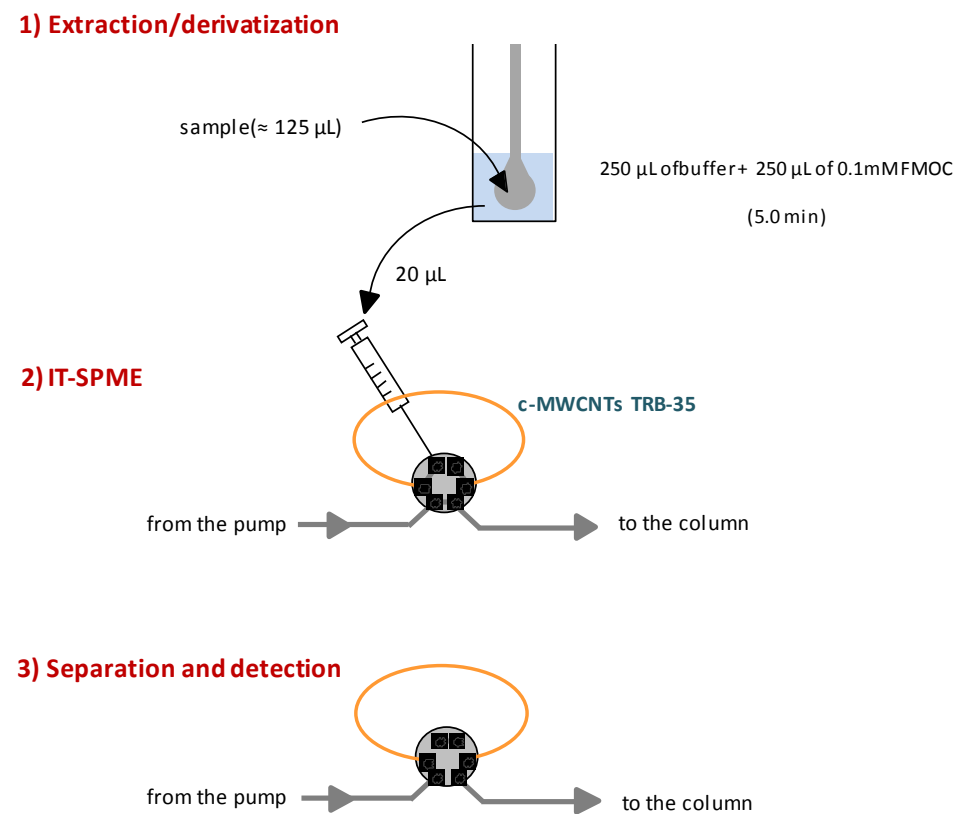

Figure 7. Scheme of the extraction/derivatization-IT-SPME-capillary liquid chromatographic method used for the analysis of amphetamines in oral fluid. The extractive capillary was a c-MWCNT functionalized TRB-35 capillary (40 cm length). For other experimental details, see text. 
In Figure 8 are shown the chromatograms obtained for a sample of oral fluid and the same sample spiked with a mixture of the analytes. The chromatograms obtained for oral fluid were similar to those obtained for standard solutions subjected to the same extraction/derivatization procedure (Figure S3). It was then concluded that the selectivity provided by the c-MWCNTs coated TRB-35 capillary was suitable. In addition, no significant differences were observed in the chromatograms obtained for samples obtained from different donors. The limits of detection (LODs), established as the concentration of amphetamines that yielded a signal-to-noise ratio of 3 , were $0.8 \mu \mathrm{g} / \mathrm{mL}$ for $\mathrm{EPE}$, and $0.5 \mu \mathrm{g} / \mathrm{mL}$ for AMP and MET. The reproducibility was also satisfactory, being the interday coefficients of variation $6 \%$ for EPE and AMP, and 3\% for MET $(n=3)$. Finally, no additional cleaning of the extractive capillary with an organic solvent after each run was necessary as during the transfer and chromatographic separation steps the capillary was flushed with an eluent containing a high percentage of acetonitrile $(70 \%)$.

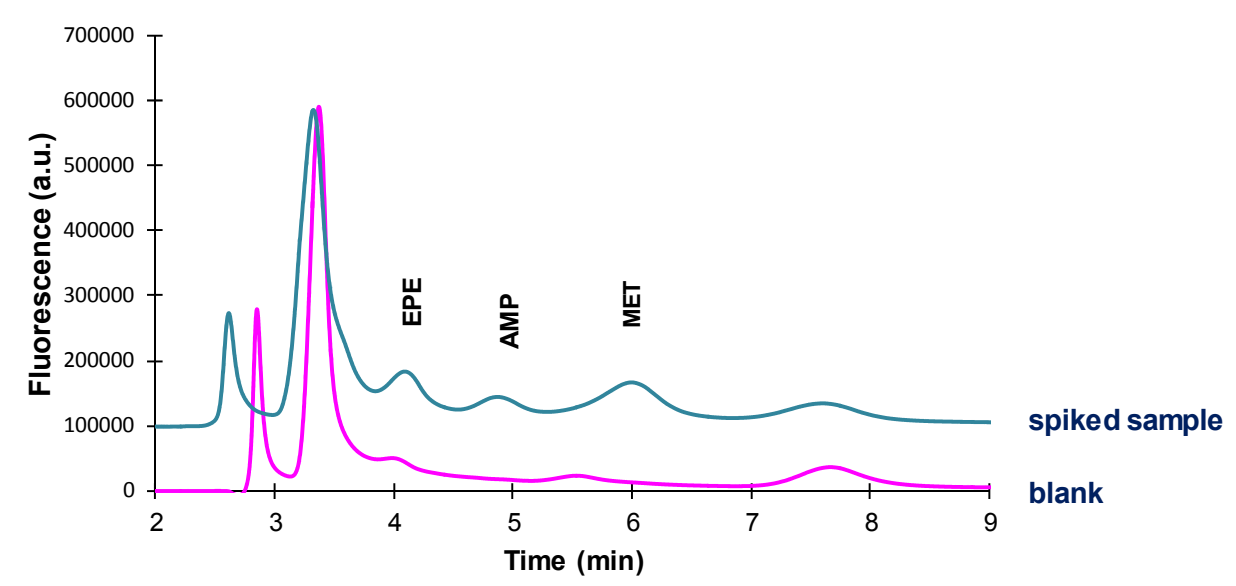

Figure 8. Chromatograms obtained with the proposed extraction/derivatization-IT-SPME-capillary liquid chromatographic method for an oral fluid sample, and the same sample spiked with the tested amphetamines $(8.0 \mu \mathrm{g} / \mathrm{mL}$, each). For other experimental details, see text.

\subsection{Utility}

Different strategies have been proposed for sample treatment prior to the chromatographic analysis of drugs in oral fluid. Extraction of the analytes into an organic solvent (toluene, butyl chloride, chloroform) has been the option traditionally used for the extraction of amphetamines $[14,18,19]$, often in combination with evaporation of the extracts to dryness in order to concentrate the extracts to be chromatographed. Solid-phase extraction followed by solvent evaporation has also been proposed [20]. These methodologies allow the detection of the analytes at low $\mathrm{ng} / \mathrm{mL}$ levels by GC, although the resulting procedures are labor-intensive. More recently, different methods have been reported that involve SPME with fibres [21,22] again prior to GC; LODs of $14-27 \mathrm{ng} / \mathrm{mL}$ were reported for MET. Very recently, microextraction with a $\mathrm{C}_{18}$ sorbent packed into the needle of a syringe has been proposed for the analysis of illicit drugs in oral fluid by LC coupled to mass spectrometry (MS) (LC-MS/MS) [23]. In this case, samples were treated to precipitate the proteins, and the supernatant was passed through the sorbent by repetitive aspirating/dispensing cycles, being the LOD reported for AMP $1 \mathrm{ng} / \mathrm{mL}$.

Compared with previously described procedures, the main advantage of the present methodology is simplicity. After sample collection, the only operations required are the immersion of the swabs into the derivatization solution and, after $5 \mathrm{~min}$, the loading of an aliquot of the resulting solution into the IT-SPME device. The proposed method is also advantageous in terms of time of analysis and solvent and reagents consumption. Although the LODs are about one order of magnitude higher than those reported by other authors, the proposed method can be considered adequate for the measurement of amphetamines in oral fluid of abusers. For example, concentrations amphetamine found in oral fluid samples during random tests programs were in the $0.3-8.8 \mu \mathrm{g} / \mathrm{mL}$ range $[24,25]$. Nevertheless, for other types of studies, more sensitive methodologies may be required. 


\section{Conclusions}

The present study shows that IT-SPME is a valid option for the on-line extraction of amphetamines derivatized with FMOC, and for their subsequent separation by capillary liquid chromatography. The efficiencies of the IT-SPME have been examined for different PDMS-based extractive capillaries, as well as for the same capillaries after their functionalization with CNTs. The introduction of CNTs in the extractive coatings has a positive effect not only on the extraction efficiencies but also on the chromatographic profiles. In all instances, highest responses were obtained for TRB-35 capillaries, which can be explained by a $\pi-\pi$ interaction mechanism. In this sense, the use of FMOC (which introduces two aromatic rings in the compounds to be extracted) may be advantageous over other derivatization reagents typically used for amphetamines [15]. On the other hand, the chromatograms observed for TRB-35 capillaries functionalized with c-SWCNTs and c-MWCNTs were quite similar, although the analytical responses obtained with the latter capillary were slightly higher. According to these results, a new approach has been described for the analysis of amphetamines derivatives using a TRB-35 capillary functionalized with c-MWCNTs.

The described approach combines the simplicity of in-valve IT-SPME with enhanced sensitivity, and can be considered an alternative for the analysis of amphetamines. As an illustrative example of application, in the present study oral fluid samples $(\approx 125 \mu \mathrm{L})$ collected with cotton swabs have directly been derivatized and processed by IT-SPME-capillary liquid chromatography. The proposed method is very simple and cost-effective, and provides suitable selectivity, sensitivity and reproducibility at low $\mu \mathrm{g} / \mathrm{mL}$ concentration levels, which are concentrations typically found in oral fluid of abusers.

Supplementary Materials: The following are available online at www.mdpi.com/2227-9075/3/1/7/s1.

Acknowledgments: The authors are grateful to the Spanish Ministerio de Economia y Competitividad/FEDER for the financial support received (project CTQ2014-53916-P) and to the Generalitat Valenciana (PROMETEO 2012/045). A. Argente-García expresses her gratitude to the Spanish Ministerio de Educación, Cultura y Deporte for her FPU grant.

Author Contributions: The manuscript was written through contributions of all authors and all authors have given approval to the final version.

Conflicts of Interest: The authors declare no conflict of interest.

\section{References}

1. Moliner-Martínez, Y.; Herráez-Hernández, R.; Verdú-Andres, J.; Molins-Legua, C.; Campíns-Falcó, P. Recent advances in in-tube solid-phase microextraction. Trends Anal. Chem. 2015, 71, 205-213. [CrossRef]

2. Kataoka, H.; Saito, K. Recent advances in SPME techniques in biomedical analysis. J. Pharm. Biomed. Anal. 2011, 54, 926-950. [CrossRef] [PubMed]

3. Queiroz, M.E.C.; Melo, M.P. Selective capillary coating materials for in-tube solid-phase microextraction coupled to liquid chromatography to determine drugs and biomarkers in biological samples: A review. Anal. Chim. Acta 2014, 826, 1-11. [CrossRef] [PubMed]

4. Mehdinia, A.; Aziz-Zanjani, M.O. Recent advances in nanomaterials utilized in fiber coatings for solid-phase microextraction. Trends Anal. Chem. 2013, 42, 205-215. [CrossRef]

5. Weng, Y.; Chen, L.; Li, J.; Liu, D.; Chen, L. Recent advances in solid-phase sorbents for samples preparation prior to chromatographic analysis. Trends Anal. Chem. 2014, 59, $26-41$.

6. González-Fuenzalida, R.A.; Moliner-Martínez, Y.; Verdú-Andrés, J.; Molins-Legua, C.; Herráez-Hernández, R.; Jornet-Martínez, N.; Campíns-Falcó, P. Microextraction with phases containing nanoparticles. Bioanalysis 2015, 17, 2163-2170. [CrossRef] [PubMed]

7. Zhang, B.-T.; Zheng, X.; Li, H.-F.; Lin, J.-M. Application of carbon-based nanomaterials in sample preparation: a review. Anal. Chim. Acta 2013, 784, 1-17. [CrossRef] [PubMed]

8. $\quad$ Liang, X.; Liu, S.; Wang, S.; Guo, Y.; Jiang, S. Carbon-based sorbents: carbon nanotubes. J. Chromatogr. A 2014, 1357, 53-67. [CrossRef] [PubMed] 
9. Liu, X.-Y.; Ji, Y.-S.; Zhang, H.-X.; Liu, M.-C. Highly sensitive analysis of substituted aniline compounds in water samples by using oxidized multiwalled carbon nanotubes as an in-tube solid-phase microextraction medium. J. Chromatogr. A 2008, 1212, 10-15. [CrossRef] [PubMed]

10. Jornet-Martínez, N.; Serra-Mora, P.; Moliner-Martínez, Y.; Herráez-Hernández, R.; Campíns-Falcó, P. Evaluation of Carbon Nanotubes Functionalized Polydimethylsiloxane Based Coatings for In-Tube Solid Phase Microextraction Coupled to Capillary Liquid Chromatography. Chromatography 2015, 2, 515-528. [CrossRef]

11. Moliner-Martínez, Y.; Serra-Mora, P.; Verdú-Andrés, J.; Herráez-Hernández, R.; Campíns-Falcó, P. Analysis of polar triazines and degradation products in waters by in-tube solid-phase microextraction and capillary chromatography: and environmentally friendly method. Anal. Bioanal. Chem. 2015, 407, 1485-1497. [CrossRef] [PubMed]

12. Samyn, L.; Laloup, M.; de Boek, G. Bioanalytical procedures for determination of drugs of abuse in oral fluid. Anal. Bioanal. Chem. 2007, 388, 1437-1453. [CrossRef] [PubMed]

13. Lledo-Fernandez, C.; Banks, C.E. An overview of quantifying and screening drugs of abuse in biological samples: Past and present. Anal. Methods 2011,3, 1227-1245. [CrossRef]

14. Kankaanpää, A.; Gunnar, T.; Ariniemi, K.; Lillsunde, P.; Mykkänen, S.; Seppälä, T. Single-step procedure for gas chromatography-mass spectrometry screening and quantitative determination of amphetamine-type stimulants and related drugs in blood, serum, oral fluid and urine samples. J. Chromatogr. B 2004, 810, 57-68. [CrossRef]

15. Herráez-Hernández, R.; Campíns-Falcó, P.; Sevillano-Cabeza, A. On-line derivatization into precolumns for the determination of drugs by liquid chromatography and column switching: determination of amphetamines in urine. Anal. Chem. 1996, 68, 734-739. [CrossRef] [PubMed]

16. Cháfer-Pericás, C.; Campíns-Falcó, P.; Herráez-Hernández, R. Application of solid-phase microextraction combined with derivatization to the determination of amphetamines by liquid chromatography. Anal. Biochem. 2004, 333, 328-335. [CrossRef] [PubMed]

17. Moliner-Martínez, Y.; Molins-Legua, C.; Verdú-Andrés, J.; Herráez-Hernández, R.; Campíns-Falcó, P. Advantages of monolithic over particulate columns for multiresidue analysis of organic pollutants by in-tube solid-phase microextraction coupled to capillary liquid chromatography. J. Chromatogr. A 2011, 1218, 6256-6262. [CrossRef] [PubMed]

18. Drummer, O.H.; Gerostamoulos, D.; Chu, M.; Swann, P.; Boorman, M.; Cairns, I. Drugs in oral fluid in randomly selected drivers. Forensic Sci. Int. 2007, 170, 105-110. [CrossRef] [PubMed]

19. Meng, P.; Wang, Y. Small volume liquid extraction of amphetamines in saliva. Forensic Sci. Int. 2010, 197, 80-84. [CrossRef] [PubMed]

20. Concheiro, M.; de Castro, A.; Quintela, O.; Cruz, A.; López-Rivadulla, M. Determination of illicit and medicinal drugs and their metabolites in oral fluid and preserved oral fluid by liquid chromatography-tandem mass spectrometry. Anal. Bioanal. Chem. 2008, 391, 2329-2338. [CrossRef] [PubMed]

21. Djozan, D.; Baheri, T. Investigation of pencil leads fiber efficiency for SPME of trace amount of methamphetamine from human saliva prior to GC-MS analysis. J. Chromatogr. Sci. 2010, 48, 224-228. [CrossRef] [PubMed]

22. Djozan, D.; Farajzadeh, M.A.; Sorouraddin, S.M.; Baheri, T. Synthesis and application of high selective monolithic fibers based on molecularly imprinted polymer for SPME of trace methamphetamine. Chromatographia 2011, 73, 975-983. [CrossRef]

23. Montesano, C.; Simeoni, M.C.; Curini, R.; Sergi, M.; lo Sterzo, C.; Compagnone, D. Determination of illicit drugs and metabolites in oral fluid by microextraction of packed sorben coupled with LC-MS/MS. Anal. Bioanal. Chem. 2015, 407, 3647-3658. [CrossRef] [PubMed]

24. Samyn, N.; van Haeren, C. On-site testing of saliva and sweat with drugwipe and determination of concentrations of drugs of abuse in saliva, plasma and urine of suspected users. Int. J. Legal Med. 2000, 113, 150-154. [CrossRef] [PubMed]

25. Samyn, N.; de Boeck, G.; Wood, M.; Lamers, C.; de Waard, D.; Brookhuis, K.; Verstraete, A.; Riedel, W. Plasma, oral fluid and sweat wipe ecstasy concentrations in controlled and real life conditions. Forensic Sci. Int. 2002, 128, 90-97. [CrossRef] 
(c) 2016 by the authors; licensee MDPI, Basel, Switzerland. This article is an open access article distributed under the terms and conditions of the Creative Commons by Attribution (CC-BY) license (http://creativecommons.org/licenses/by/4.0/). 